\title{
ON THE AUTOMORPHISM GROUP OF A SEMISIMPLE JORDAN ALGEBRA OF CHARACTERISTIC ZERO
}

\author{
BY S. ROBERT GORDON ${ }^{1}$
}

\author{
Communicated by S. Lang, November 14, 1968
}

Introduction. Let $\Im$ be a semisimple Jordan algebra over an algebraically closed field $\Phi$ of characteristic zero, and let $G$ be the automorphism group of $\Im$. The purpose of this note is to present general results on $G$, the proofs of which do not involve the use of the classification theory of simple Jordan algebras over $\Phi$. Specifically, we wish to determine the algebraic components $G_{0}, G_{1}, G_{2}, \ldots$ of the linear algebraic group $G$. To this end, we will give a formula for the number of components of $G$ in terms of certain root-spaces associated with $\Im$ (see the Corollary to Theorem 3 and Theorem 6 below). For each component $G_{i}$ of $G$, the index of $G_{i}$ is defined to be the minimum dimension of the 1-eigenspaces of the automorphisms belonging to $G_{i}$. We will give a formula for the index of each component $G_{i}$ of $\Im$ (see Theorem 8). Finally, we will give a table which applies these theorems to each of the simple Jordan algebras over $\Phi$.

These results are analogous to those on Lie algebras given in [4, Chapter 9] and [5].

1. Notation and terminology. Let $\Im, G$, and $\Phi$ be as above. Following [3], we write $x . y$ for the product of elements $x, y$ of $\Im$ and let $R_{y}: x \rightarrow x . y$. We let $\mathfrak{D}$ be the derivation algebra of $\Im$. We denote by $\mathfrak{i}$ the structure Lie algebra $R_{\Im} \oplus \mathfrak{D}$ of $\Im$, and by $\Re$ the Koecher-Tits algebra $\Im \oplus \bar{\Im} \oplus \mathbb{R}$ of $\Im[3$, Chapter 8$]$. $\mathfrak{D}$ and $\mathfrak{R}$ are completely reducible. Thus if $\mathfrak{E}$ is the center of $\mathbb{R}$ and $\mathbb{E}$ the center of $\mathfrak{D}$, then $\mathbb{R}=\mathfrak{C} \oplus \mathfrak{R}^{\prime}$ and $\mathfrak{D}=\mathfrak{E} \oplus \mathfrak{D}^{\prime}$, where $\mathfrak{R}^{\prime}$ and $\mathfrak{D}^{\prime}$ are semisimple. $\mathbb{R}$ is semisimple and is simple if and only if $\Im$ is simple. Let $\Gamma$ be the structure group of $\Im$ [3, Chapter 2]. $G$ and $\Gamma$ are linear algebraic groups; we let $G_{0}$ and $\Gamma_{0}$ be respectively the algebraic components of the identity of these groups. If $\eta \in \Gamma$, then $\tilde{\eta}: a+b+L \rightarrow a \eta+\left(b \eta^{k-1}\right)-+\eta^{-1} L \eta$ is an automorphism of $\Omega$; here $\eta^{\sharp}=U_{1 \eta} \eta^{-1}$, where in general $U_{x}=2 R_{x}^{2}-R_{x} \cdot s$ (see [6]). The mapping $\eta \mapsto \tilde{\eta}$ is a birational isomorphism from $\Gamma$ onto a subgroup $\tilde{\Gamma}$ of Aut $\Omega$. $\tilde{\Gamma}$ is the subgroup of Aut $\Omega$ of elements fixing $R_{1}$ (where 1 is the identity of $\Im$ ).

1 These results are contained in the author's doctoral thesis, written at Yale University under the direction of Professor N. Jacobson. The research was supported in part by an NSF Graduate Fellowship and in part by NSF grant GP-8023. 
Let $\mathfrak{W}$ be a fixed Cartan subalgebra of $\mathfrak{D}$. Then $\mathfrak{W}=\mathbb{E} \oplus \mathfrak{W}_{1}$, where $\mathfrak{W}_{1}=\mathfrak{W} \cap \mathfrak{R}^{\prime}$ is a Cartan subalgebra of $\mathfrak{R}^{\prime}$. Let $\mathfrak{A}=\{x \in \mathfrak{Y} \mid a \mathfrak{W}=0\}$. $\mathfrak{E}=R \mathfrak{R}+\mathfrak{W}$ is the unique Cartan subalgebra of $\mathbb{R}$ containing $\mathfrak{W}$, and any Cartan subalgebra of $\mathfrak{R}$ is a Cartan subalgebra of $\Omega$. If $\mathfrak{W}_{1}=\bigvee_{\mathscr{Z}} \cap \mathfrak{R}^{\prime}$, then $\mathfrak{W}_{1}$ is a Cartan subalgebra of $\mathbb{Z}^{\prime}$ and $\mathfrak{W}=\mathfrak{S} \oplus \mathfrak{L}_{1}$. We let $($,$) denote$ the Killing form of $\Omega$ and also the nondegenerate symmetric bilinear form on $\mathfrak{W}^{*}$ induced by the Killing form of $\Re$. Similarly, we let $\langle$, denote the Killing form of $\mathbb{Z}^{\prime}$ and the corresponding form on $\mathfrak{W}_{1}{ }^{*}$.

2. Roots and root spaces. Let $\alpha \rightarrow \hat{\alpha}$ be the linear transformation from $\mathfrak{S}_{1}^{*}$ to $\mathfrak{S}^{*}$ which is the dual of the natural projection of $\mathfrak{S}=\mathfrak{C} \oplus \mathfrak{S}_{1}$ onto $\mathfrak{W}_{1}$.

THEOREM 1. If $\rho$ is a root of $\Omega$ then $\rho\left(R_{1}\right)=+1,-1$, or 0 according as the root space $\Omega_{\rho}$ belongs to $\Im, \bar{\Im}$, or $\mathfrak{R}^{\prime}$. If $\alpha$ is a root of $\mathfrak{R}^{\prime}$ then $\hat{\alpha}$ is a root of $\Omega$. The roots of $\Omega$ of the form $\hat{\alpha}\left(\alpha\right.$ a root of $\left.\Omega^{\prime}\right)$ are exactly the roots $\rho$ such that $\rho\left(R_{1}\right)=0$. If $\alpha, \beta$ are two roots of $\mathfrak{R}^{\prime}$ then $2\langle\alpha, \beta\rangle /\langle\alpha, \alpha\rangle$ $=2(\hat{\alpha}, \hat{\beta}) /(\hat{\alpha}, \hat{\alpha})$. If $\alpha_{1}, \cdots, \alpha_{l}$ is a simple system of roots of $\mathbb{R}^{\prime}$, then there is a unique set $\left\{\rho_{1}, \cdots, \rho_{r}\right\}$ of roots of $\AA$ such that $\rho_{1}\left(R_{1}\right)=\ldots$ $=\rho_{r}\left(R_{1}\right)=1$ and $\left\{\rho_{1}, \cdots, \rho_{r}, \hat{Q}_{1}, \cdots, \hat{Q}_{l}\right\}$ is a simple system of roots of $\Omega$.

The mapping $\epsilon: a+\bar{b}+R_{c}+D \rightarrow b+\bar{a}-R_{\mathrm{c}}+D(a, b, c \in \Im, D \in \mathfrak{D})$ is an automorphism of $\mathfrak{R}$. It stabilizes $\mathfrak{R}, \mathfrak{L}^{\prime}, \mathfrak{C}, \mathfrak{W}$, and $\mathfrak{W}_{1}$; we let $\epsilon^{*}$ denote the dual transformation both of $\mathfrak{W}^{*}$ and $\mathfrak{W}_{1}^{*}$. $\mathfrak{W}_{1}^{*}$ is the direct sum of the subspaces $\left\{\alpha \in \bigvee_{1}^{*} \mid \alpha \epsilon^{*}=-\alpha\right\}$ and $\left\{\alpha \in \bigvee_{1}^{*} \mid \alpha \epsilon^{*}=\alpha\right\}$, and these subspaces are orthogonal with respect to $\langle$,$\rangle . The second sub-$ space can be identified in a natural way with $\mathfrak{B}^{*}$. For $\alpha \in \mathfrak{W}_{1}^{*}$, let $\alpha_{+}$ be the projection of $\alpha$ onto $\mathfrak{W}^{*}$. It can be seen that $\epsilon^{*}$ stabilizes some simple system of roots of $\mathfrak{L}^{\prime}$. In this way $\epsilon^{*}$ induces an automorphism of the Dynkin diagram of $\mathfrak{Z}^{\prime}$. We can therefore apply [8, Theorem 32] to conclude that $\left\{\alpha_{+} \mid \alpha\right.$ is a root of $\left.\mathfrak{R}^{\prime}\right\}$ is a (not necessarily reduced) root system, which we call $\Sigma_{\epsilon}$.

THEOREM 2. Let $\alpha$ be a root of $\mathfrak{R}^{\prime}$ and let $R_{a}+B$ be a root vector for $\alpha$. Then $B$ is nonzero if and only if $\alpha \mid \mathfrak{W}_{1}$ is a root of $\mathfrak{D}^{\prime}$; in this case $\alpha(\mathbb{E})=0$ and $\alpha \mid \mathfrak{W}_{1}$ has root vector $B$. If $\omega$ is a root of $\mathfrak{D}^{\prime}$ then there is a root $\beta$ of $\mathfrak{R}^{\prime}$ with root vector $R_{a}+B, B \neq 0$, such that $\beta \mid \mathfrak{W}_{1}=\omega$.

This theorem allows us to identify the roots of $\mathfrak{D}^{\prime}$ with a subset of $\Sigma_{\text {. }}$. Thus if $\alpha$ is a root of $\mathfrak{R}^{\prime}$ and $\alpha \mid \mathfrak{W}_{1}=\omega$ is a root of $\mathfrak{D}^{\prime}$, we identity $\omega$ with $\alpha_{+}$. If $\omega, \psi$ are roots of $\mathfrak{D}^{\prime}$ identified respectively with $\alpha_{+}$and $\beta_{+}$ and if $w_{\psi}$ and $w_{\beta_{+}}$are the reflections of the appropriate root spaces in 
the directions of $\psi$ and $\beta_{+}$respectively, then it can be shown that $\alpha_{+} w_{\beta_{+}}$is identified with $\omega w_{\psi}$. This means that the Weyl group of $\mathfrak{D}^{\prime}$ can be embedded in a natural way in the Weyl group of $\Sigma_{\text {e. }}$

3. Automorphisms. From now on we assume we have a fixed simple system $\alpha_{1}, \cdots, \alpha_{l}$ of roots of $\mathfrak{R}^{\prime}$ stabilized by $\epsilon^{*}$. We let

$$
\left\{\rho_{1}, \cdots, \rho_{r}, \hat{\alpha}_{1}, \cdots, \hat{\alpha}_{l}\right\}
$$

be the corresponding simple system of roots of $\Omega$, as in Theorem 1 .

THEOREM 3. (a) If $\eta \in \tilde{\Gamma}$ then there exists $a \tau \in \tilde{\Gamma}_{0}$ so that $\eta \tau$ stabilizes $\mathfrak{S}$.

(b) If $\eta \in$ Aut $\Re$ and $\eta$ stabilizes $\mathfrak{Q}$, then $\eta \in \tilde{\Gamma}$ if and only $\eta^{*}$ (the dual transformation of $\eta \mid \mathfrak{E}$; note that $\eta^{*}$ permutes the roots of $\Re$ ) permutes the roots $\rho$ of $\Omega$ such that $\rho\left(R_{1}\right)=1$.

(c) If $\eta \in \tilde{\Gamma}$ stabilizes $\mathfrak{S}$, then $\eta \in \tilde{\Gamma}_{0}$ if and only if $\eta^{*}$ is in the Weyl group of $\Omega$.

CoRollary. Each algebraic component of Aut $\Re$ contains at most one component of $\tilde{\Gamma}$. It contains exactly one if and only if the corresponding automorphism of the Dynkin diagram of $\Omega$ permutes the points corresponding to $\rho_{1}, \cdots, \rho_{r}$. Thus $\left[\Gamma: \Gamma_{0}\right]$ is the number of such automorphisms of the diagram of $\Omega$.

THEOREM 4. (a) If $\eta \in G$, there exists $\tau \in G_{0}$ so that $\eta \tau$ stabilizes $\mathfrak{W}$.

(b) If $\eta \in G$ stabilizes $\mathfrak{G}$, then $\eta^{*}$ (acting in $\mathfrak{S}^{*}$ ) permutes the roots $\rho$ of $\Re$ such that $\rho\left(R_{1}\right)=1$ and commutes with $\epsilon^{*}$. Conversely, if $w$ is a linear transformation of $\mathfrak{\Phi}^{*}$ which permutes the roots of $\Re$, permutes the roots $\rho$ such that $\rho\left(R_{1}\right)=1$, and commutes with $\epsilon^{*}$, then there is a $\zeta \in G$ such that $\bar{\zeta}$ stabilizes $\mathfrak{S}$ and $\bar{\zeta}^{*}=w$.

TheOREM 5. Let $\eta \in G$. Necessary and sufficient conditions for $\eta$ to be in $G_{0}$ are that

(i) $\eta$ commutes with any $E \in \mathbb{E}$,

(ii) $\tilde{\eta} \mid \mathfrak{D}^{\prime}$ is in the component of the identity of Aut $\mathfrak{D}^{\prime}$,

(iii) $\bar{\eta}$ is in the component of the identity of Aut $\Omega$.

ThEOREM 6. The number of components of $G$ is the number of components of $\Gamma$ times the index of the Weyl group of $\mathfrak{D}^{\prime}$ in the Weyl group of $\Sigma_{\text {. }}$

We also see from Theorem 5 that a component of $G$ is specified by giving the corresponding action on together with the corresponding automorphisms of the Dynkin diagrams of $\Re$ and $\mathfrak{D}^{\prime}$. 
4. Fixed points. If $\mathfrak{B}$ is a vector space over $\Phi, \lambda \in \Phi$, and $\eta \in \operatorname{Hom}_{\Phi}(\mathfrak{B}, \mathfrak{B})$ then by $\mathfrak{B}_{\lambda}(\eta)$ we mean the $\lambda$-eigenspace of $\eta$. If $G_{i}$ is a component of $G$, the index of $G_{i}$ is defined to be the minimum dimension of $\Im_{1}(\eta)$ for $\eta \in G_{i}$; if $\eta \in G_{i}, \eta$ is said to be regular if $\operatorname{dim} \Im_{1}(\eta)$ equals the index of $G_{i}$.

THEOREM 7. If $\eta \in G$ then $\Im_{1}(\eta)$ is a semisimple subalgebra of $\Im$. The automorphism $\eta$ is regular if and only if $\Im_{1}(\eta)$ is associative (i.e., is a direct sum of fields). If $\eta$ is regular then $\eta$ fixes $\Im_{1}(\eta)$ pointwise.

Corollary. The index of $G_{i}$ is also the minimum dimension of the fixed point spaces of automorphisms in $G_{i}$.

THEOREM 8. Let $G_{i}$ be a component of $G$. Let $N$ be the index of $G_{i}$. Let $M$ be the index of the component of Aut $\Omega$ to which $\tilde{G}_{i}$ belongs. Let $P$ be the index of the component of Aut $\mathfrak{D}^{\prime}$ to which $\widetilde{G}_{i} \mid \mathfrak{D}^{\prime}$ belongs. Since for $\zeta \in G_{i}, \tilde{\zeta} \mid \mathbb{F}$ is independent of $\zeta$ (by Theorem $5(\mathrm{i})$ ), we can let $Q$ be the dimension of $\mathbb{E}_{1}(\tilde{\zeta} \mid \mathbb{E})$ for $\zeta \in G_{i}$. Then $N=M-P-Q$.

Corollary. The index of $G_{0}$ is rank $\Re$-rank $\mathfrak{D}$.

THEOREM 9. The minimum dimension of the kernel of a derivation of $\Im$ is the same as the minimum dimension(for all derivations $D$ ) of $\Im_{0}(D)$. This number is rank $\Re$ - rank $\mathfrak{D}$.

5. Examples. We recall the classification of simple Jordan algebras over $\Phi$ (see [3]). Any finite-dimensional simple algebra is isomorphic to one of the following.

(i) $\Phi 1 \oplus \mathfrak{B}$, the Jordan algebra of a vector space $\mathfrak{B}$ of dimension at least 2, equipped with a nondegenerate symmetric bilinear form; $\Phi$;

(ii) $\mathfrak{S}\left(\Phi_{n}\right)$, the Jordan algebra of all symmetric $n \times n$ matrices over

(iii) $\Phi_{n}^{+}$, the Jordan algebra of all $n \times n$ matrices over $\Phi$;

(iv) $\mathfrak{S}\left(\Phi_{2 n}, J_{S}\right)$, the algebra of all $2 n \times 2 n$ matrices symmetric with respect to a skew bilinear form;

(v) $\mathfrak{S}\left(\mathfrak{D}_{8}\right)$, the set of all $3 \times 3$ hermetian matrices over the Cayley algebra $\mathfrak{O}$.

For all these algebras except $\Phi 1 \oplus \mathfrak{B}, \operatorname{dim} \mathfrak{B}=2, \mathfrak{D}$ is semisimple $[1]$; i.e., $\mathbb{E}=0$. In this case the components are uniquely specified by the corresponding automorphisms of the Dynkin diagrams of $\Omega$ and $\mathfrak{D}$. The accompanying table shows how these theorems apply to each of the components of $G$ for each of these algebras. The circled points in the diagrams of $\Re$ are the points corresponding to the simple roots $\rho_{1}, \cdots, \rho_{r}$. 
I969] SEMISIMPLE JORDAN ALGEBRA OF CHARACTERISTIC ZERO

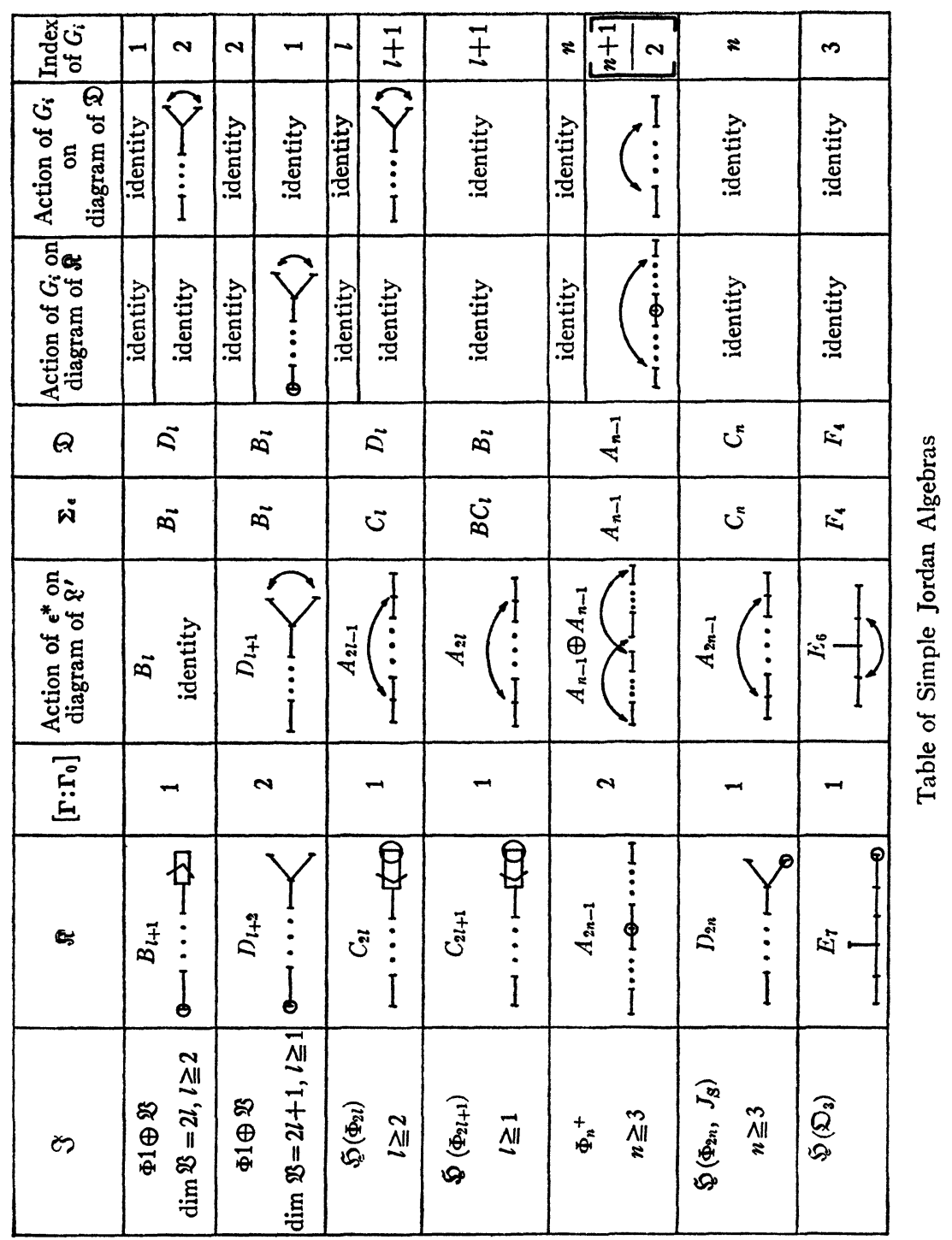




\section{REFERENCES}

1. Karl-Heinz Helwig, Über Automorphismen und Derivationen von Jordan Algebren, Nederl. Akad. Wetensch. Proc. Ser A 70 (1967), 381-394.

2. - Halbeinfache Reele Jordan-Algebren, Habilitationsschrift, University of Munich, München, 1967.

3. N. Jacobson, Structure and representations of Jordan algebras, Amer. Math. Soc. Colloq. Publ., vol. 39, Amer. Math. Soc., Providence, R. I., 1969.

4. - Lie algebras, Interscience, New York, 1962.

5. - A note on automorphisms of Lie algebras, Pacific J. Math 12 (1962), 303-315.

6. M. Koecher, On Lie algebras defined by Jordan algebras, Mimeographed Notes, Århus Universitet, 1967.

7. J.-P. Serre, Algebres de Lie semi-simples complexes, Benjamin, New York, 1966.

8. R. Steinberg, Lectures on Chevalley groups, Mimeographed Notes, Yale University, New Haven, Conn., 1967.

Yale University, New Haven, Connecticut 06520 